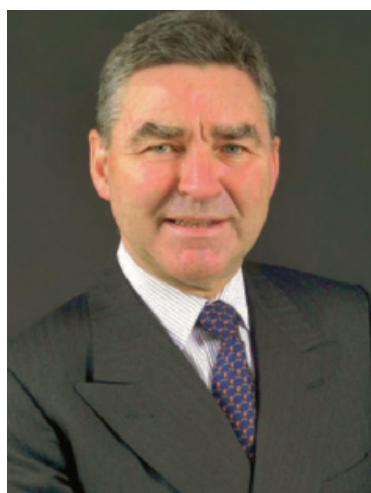

A. Crockard

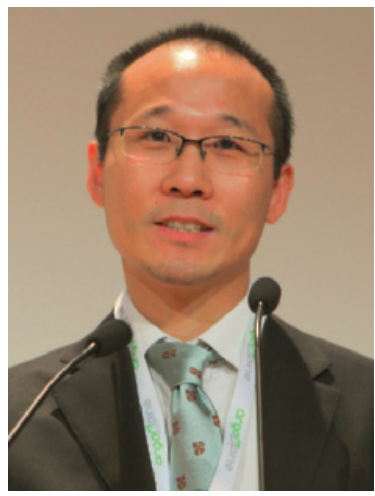

D. Choi

\section{Overview of cerebrospinal fluid leakage}

Since the days of the ancient Pharaohs, leakage of cerebrospinal fluid has been known to have potentially fatal consequences and should not be underestimated. The purpose of this special supplement is to draw spinal surgeons' attention to the clinical history, investigation and treatment of CSF leaks.

Brisby et al. focus on CSF leakage during or after elective surgery for spinal disc disease. They point out that the reported incidence is as high as $16 \%$ in elective surgery, and dural tears are more common in elderly patients, lumbar surgery, ossification of the posterior longitudinal ligament, and when surgeons are less experienced. Massicotte and George add to this list of risk factors for CSF leakage, including the connective tissue disorders such as Marfans and Ehlers Danlos syndromes. In their article they define the terms: durotomy, CSF leak, pseudomeningocoele, and duro-cutaneous fistula.

Choi highlights that not all CSF leaks are complications of surgery or due to surgeon error. During surgery for spinal tumours for example, often the dura is purposely resected for complete tumour clearance. These wide resections of dura can be a challenge to repair, and pre-operative planning is essential to prevent complications. Surgeons should remember that operations for spinal tumours usually aim to improve quality of life and relieve symptoms; these goals can be easily negated by the complications of CSF leakage.

Depreitere et al. comment that most dural tears are noticed at the time of surgery, and therefore immediate and adequate primary closure of the CSF leak is important. Although there are many commercial products, glues and sealants available, the most efficient method for stopping a CSF leak is by accurate closure by primary suture. There is no clear consensus on when or whether to use an intrathecal lumbar CSF drain, a superficial wound drain, or no drain at all. Some authors advocate lumbar CSF drainage to allow the primary closure to heal adequately, whereas others suggest that lumbar drainage itself can add to the complications of low CSF pressure and infection. This debate will continue until scientific evidence from randomised trials becomes available.

Styliaras et al. discuss the difficulties in diagnosis and management of CSF leaks after penetrating injuries caused by knives and bullets. For many, the escape of CSF through an open skin wound may be controlled by 96 hours of bed-rest, and a contained meningocoele in a closed injury may be observed by follow-up imaging in many circumstances. High velocity and impact battlefield injuries represent a more serious problem where the effects of the blast and multiple organ involvement are a priority.

Each chapter contains a very useful list of references to help the serious reader to enquire further. Choi, Singh and Wang provide clinical examples - so often we learn more easily by following examples of patient management, rather than from tables and flowcharts. There is some repetition in the detail of these chapters, particularly in the diagnosis and treatment of CSF leakage, but this is intentional for two reasons: firstly to reinforce the message of this special supplement, and secondly, there is no gold-standard, evidence-based method for dealing with CSF leaks, and one-size certainly does not fit all. The reader will see that there are some common threads in the management plans discussed, and some differences in opinion, which will together allow the reader to formulate their own opinion on how to avoid, detect, and treat this potential complication. Our overall aim is to alert all spinal surgeons to this uncommon but important complication of open and minimally invasive surgery. Early diagnosis is paramount, and repair requires good microsurgical technique.

A. Crockard ${ }^{1}$, D. Choi ${ }^{2}$ 